\title{
Open versus Closed Surgery for Axillary Osmidrosis: A Meta-Analysis of Articles Published in Four Languages
}

\author{
Misako Nomura, Daichi Morioka, Yasutaka Kojima, Ryutaro Tanaka, Koichi Kadomatsu \\ Department of Plastic Surgery, Showa University, Tokyo, Japan
}

Background: Individuals with axillary osmidrosis suffer detrimental effects to their psychosocial functioning. In Asian nations, major operations for axillary osmidrosis include subdermal excision (open surgery) and suction-curettage (closed surgery). Objective: The aim of this meta-analysis was to determine which of these two procedures is most favorable in terms of safety and efficacy. Methods: According to the Preferred Reporting Items for Systematic Reviews and Metaanalyses (PRISMA) guideline, we searched electronic databases for articles published in English, Japanese, Korean, and Chinese languages. Fixed-effects model meta-analyses of odds ratios $(\mathrm{OR})$ and $95 \%$ confidence intervals $(\mathrm{Cl})$ were conducted, and the $\mathrm{I}^{2}$ was used to assess heterogeneity. Complication rates, recurrence/ineffectiveness rates, and patient satisfaction data were extracted and compared between open and closed surgeries. Results: Our search yielded 8 articles that include 1,179 patients; 560 underwent open surgery, and 619 underwent closed surgery. Our meta-analysis revealed that suction-curettage had a significantly lower risk of acute adverse events than open excision $(\mathrm{OR}, 0.15 ; 95 \% \mathrm{Cl}$, $0.07 \sim 0.32$ ), whereas open excision was significantly superior to suction-curettage for recurrence/ineffectiveness rate (OR, 2.90; 95\% Cl, $1.37 \sim 6.15)$. Patient satisfaction was equally high with both treatments $(\mathrm{OR}, 1.58 ; 95 \% \mathrm{Cl}, 0.69 \sim$

Received February 5, 2020, Revised May 19, 2020, Accepted for publication June 17, 2020

Corresponding author: Daichi Morioka, Department of Plastic Surgery, Showa University, 1-5-8 Hatanodai, Shinagawa-ku, Tokyo 142-8866, Japan. Tel: 813-3784-8548, Fax: 81-3-3784-9183, E-mail: dmorioka@gmail.com ORCID: https://orcid.org/0000-0002-6210-9808

This is an Open Access article distributed under the terms of the Creative Commons Attribution Non-Commercial License (http://creativecommons. org/licenses/by-nc/4.0) which permits unrestricted non-commercial use, distribution, and reproduction in any medium, provided the original work is properly cited.

Copyright (c) The Korean Dermatological Association and The Korean Society for Investigative Dermatology
3.60). Conclusion: Since surgical treatments for axillary osmidrosis have been performed mostly in East Asian nations, it was meaningful to review articles published in four languages. This meta-analysis revealed that closed surgery was safer but less effective than open surgery. However, both patient groups expressed high satisfaction with the outcomes. Our results may be helpful for deciding surgical treatment options. (Ann Dermatol 32(6) 487 495, 2020)

\section{-Keywords-}

Axillary osmidrosis, Complications, Meta-analysis, Surgery, Systematic review

\section{INTRODUCTION}

Axillary osmidrosis is characterized by malodor resulting from bacterial decomposition of secretions from the axillary apocrine glands ${ }^{1}$. Especially in East Asian nations, individuals with axillary osmidrosis suffer detrimental effects to their psychosocial functioning because of the offensive odor $^{1,2}$.

A wide variety of treatment options for axillary osmidrosis have been reported, mostly from East Asian countries ${ }^{2,3}$. Nonsurgical treatments include the use of topical deodorant and subcutaneous injection of botulinum toxin- $\mathrm{A}^{2}$. Surgical treatments include apocrine gland destruction by lasers, suction-curettage, and conventional open excision of the apocrine glands ${ }^{2-6}$.

In general, nonsurgical treatments and lasers are preferred for individuals with mild osmidrosis ${ }^{2}$. For moderate to severe cases, however, the main factors to consider when deciding among treatment options include adverse events, rates of recurrence, and efficacy. Open surgery is thought to be more effective based on lower recurrence rates, although complication rates are high $^{2,5}$. Introduced to Japan 
in the 1950's, open excision of the apocrine glands is usually performed ${ }^{7}$. It is common, because the entire procedure can be performed using basic surgical instruments, and it is cost-effective compared to other treatment options $^{2}$. On the other hand, since suction-curettage was first introduced by Taiwanese surgeons in 1998, it has become popular among Asian countries, and it has been modified by many surgeons ${ }^{8}$. Currently, subdermal excision as an open surgery and suction-curettage as a closed surgery are two major operations for axillary osmidrosis.

To our knowledge, two meta-analyses of the treatment of axillary osmidrosis have been published recently, and the safety and efficacy of open versus closed surgery were compared $^{5,6}$. However, case series and uncontrolled observations were included in the systematic reviews ${ }^{5}$, or various types of open surgery and nonsurgical treatments were included in the control groups ${ }^{6}$.

We therefore attempted to revisit the topics of the previously published systematic reviews and meta-analyses of surgical treatments for axillary osmidrosis and identify the comparative safety and efficacy of open versus closed surgery.

\section{MATERIALS AND METHODS}

A systematic literature search was performed between 1 and 3 October 2019. Systematic review and meta-analysis were planned, conducted, and reported in accordance with the guidelines of the Preferred Reporting Items for Systematic Reviews and Meta-analyses (PRISMA) guidelines ${ }^{9}$. No ethical committee approval was needed, because this meta-analysis was based only on previously published articles.

\section{Search strategy}

We searched the Cochrane Library, PubMed and Scopus databases to identify English and other-language articles. We also searched Ichushi-Web and KoreaMed to identify Japanese and Korean language articles, respectively. The China National Knowledge Infrastructure (CNKI) and Taiwan periodical literature system (PerioPath) were used to search for published literature in Chinese languages. In addition, all references cited in the selected articles were hand-searched to identify articles that were not indexed by the electronic databases.

The following combinations of search terms were used: ("osmidrosis" or "bromidrosis" or "bromhidrosis" or "hircismus") and ("surgery" or "surgical treatment"). Keywords corresponding to those terms were used for non-English databases (e,g. "ekisyusho" for osmidrosis in Japanese language).

\section{Study selection and inclusion criteria}

The screening process was performed independently by two of the authors ( $\mathrm{MN}$ and DM). Interrater reliability assessed by a kappa statistic was 0.826 (excellent reproducibility). Any disagreement was resolved by consensus. The inclusion criteria of this meta-analysis were prospective or retrospective cohort studies or observational studies that focused on comparison of long-term outcomes and recurrence or efficacy between open and closed surgeries, described in one of four languages (English, Japanese, Korean, or Chinese) and published in 2001 or later. Short communications meeting these inclusion criteria with sufficient numbers of subjects and lengths of follow-up periods were also included.

Open surgery, defined as the control procedure in this meta-analysis, was conventional open excision of the subdermis including the apocrine glands from a 2 to $6 \mathrm{~cm}$ single skin incision along the axillar crease. This procedure was performed with the use of a basic set of surgical instruments, including forceps, skin hooks and dissecting scissors. Bleeding was usually controlled by electric cauterization.

Closed surgery, defined as an experimental procedure, was curettage of subdermal tissues through small skin incisions (less than $1 \mathrm{~cm}$ ). This procedure was performed blindly with the use of special curettage cannulas, curettes or electric shavers provided with a suction system. Bleeding was controlled by drainage tubes and compression dressings.

\section{Exclusion criteria}

Studies using open excision from multiple skin incisions, closed surgery using a blunt liposuction cannula without curettage, and combination treatments with other apparatuses, such as an ultrasonic aspirator and laser, were excluded. Studies with insufficient numbers of subjects (less than 30) were also excluded. In addition, articles with no English title and abstract were excluded.

\section{Data extraction}

Two authors ( $\mathrm{MN}$ and $\mathrm{DM}$ ) independently extracted the relevant data from the selected studies. Any disagreements were resolved by discussion, and a final decision was made by consensus.

Outcome measures investigated were the complication rate (as a safety measure) and the recurrence or ineffectiveness rate (as an efficacy measure). Complications were limited to acute, moderate to severe adverse events, which included hematoma, seroma, infection, skin necrosis, wound dehiscence, and nerve injury. Mild adverse events, such 
as contact dermatitis from dressing tape and transient erythema, were excluded. Long-lasting or delayed complications, such as scar contracture, keloids, hyperpigmentation and epidermal inclusion cysts, were also excluded from the present analysis.

Recurrence and ineffectiveness were assessed by the physicians or patients themselves. Descriptions such as "not improved" or "not effective" as well as "recurrence" accounted for the rates in this analysis.

We also assessed patient satisfaction as an indicator of efficacy. Satisfaction in this analysis included "fully satisfied" to "fairly satisfied;" "poorly satisfied" and "not satisfied" were not included.

\section{Risk-of-bias assessment}

A modified Newcastle-Ottawa scale (maximum score of 7) was used to evaluate the methodological quality of all included studies ${ }^{10}$. High quality was defined as a score of $\geq 5$, while low quality was defined as a score of $\leq 4$. Quality was independently assessed by two authors (MN and DM) for English, Korean, and Japanese language studies, and by an author (DM) and a coauthor (LL) for Chinese language studies. Any discrepancies in quality assessment were discussed until a consensus was reached.

\section{Statistical analysis}

Statistical analyses were performed with Review Manager Software ver. 5.3 (RevMan v5.3; The Cochrane Collaboration, Oxford, UK). A fixed-effects model using the MantelHaenszel method was employed to account for heterogeneity. Heterogeneity was assessed using I $\mathrm{I}^{2}$ tests. An I $\mathrm{I}^{2}$ greater than $50 \%$ indicated significant heterogeneity. Odds ratios (OR) and 95\% confidence intervals $(95 \% \mathrm{Cl})$ were used for dichotomous outcomes of safety and efficacy. Forest plots were used to display the effect size of each study graphically. Values of $p<0.05$ between groups were considered statistically significant.
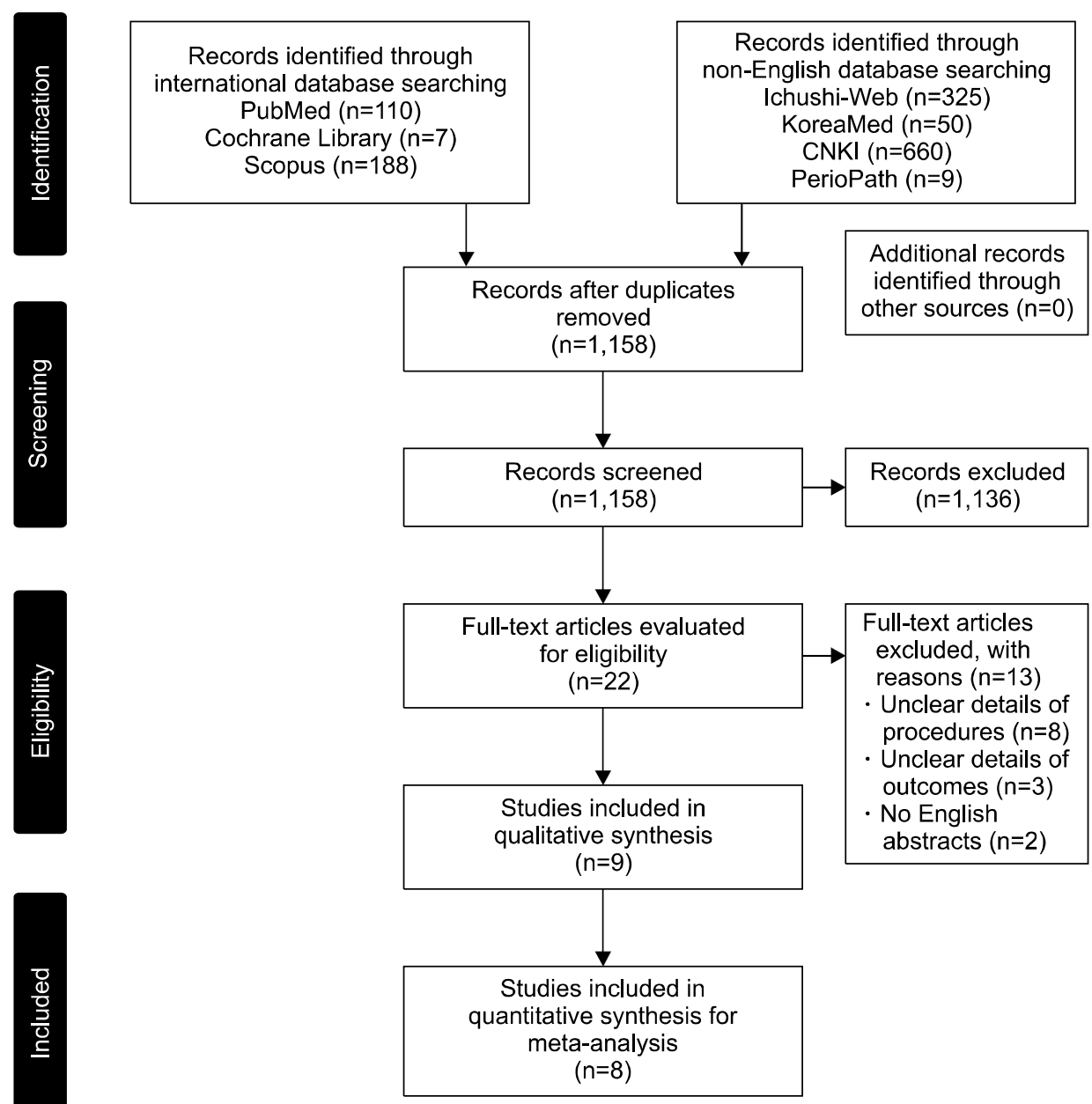

Fig. 1. The Preferred Reporting Items for Systematic Reviews and Metaanalyses (PRISMA) flowchart of literature review process. 


\section{RESULTS}

\section{Study selection}

Overall, our literature search strategies identified 1,349 articles (110 through PubMed, 7 through the Cochrane Library, 188 through Scopus, 325 through Ichushi-Web, 50 through KoreaMed, 660 through CNKI, and 9 through PerioPath). After duplicates were removed, 1,158 potentially relevant articles were screened. No article was identified through manual searching of abstracts. After screening titles and abstracts, 22 articles were retrieved for full text evaluation, and 9 articles satisfied the inclusion criteria of qualitative synthesis. One of these studies was excluded because of a very low quality score, and finally, 8 articles were included in this meta-analysis. Because the number of included studies was smaller than 10, publication bias was not evaluated. The selection and screening process is summarized in Fig. 1.

\section{Study characteristics}

Detailed characteristics of the patients are summarized in Table $1^{3,11-17}$. The database search yielded 8 articles that included 1,179 patients. A total of 560 patients (1,120 axillae) underwent open surgery, and 619 patients $(1,238$ axillae) underwent closed surgery. Of these 8 articles ${ }^{3,11-17}$, five were published in Chinese; one article (study performed in China) was published in English, one in Japanese and one in Korean.

The minimum follow-up period was 3 months in three studies $^{3,11,13}, 6$ months in four studies ${ }^{12,14,16,17}$, and unclear in one study ${ }^{15}$. Four articles matched demographics between groups of subjects (male to female ratio and mean age $)^{3,11,14,17}$, whereas two studies did not match the male to female ratio of each group ${ }^{13,15}$, and two studies described neither gender nor age of the subjects in each group $^{12,16}$. Consequently, four studies ${ }^{12,13,15,16}$ were assessed as having a low risk of bias, and four were assessed as having a high risk of bias.

\section{Complications}

The eight studies qualitatively synthesized in the systematic review were included in the meta-analysis. However, one study by Li et al. ${ }^{12}$ was excluded from the present meta-analysis of the complication rate, because details of adverse events were missing. Forest plots are shown in Fig. $2^{3,11,13-17}$. There was no heterogeneity with respect to acute adverse events, as reflected by an $\mathrm{I}^{2}$ of $19 \%$. The meta-analysis revealed that suction-curettage was associated with a significantly lower risk of acute adverse events than open excision $(\mathrm{OR}, 0.15 ; 95 \% \mathrm{Cl}, 0.07$ 0.32 ; Fig. 2A). Three studies ${ }^{3,11,17}$ that evaluated complications by axilla were analyzed separately, and the metaanalysis revealed similar results $\left(\mathrm{I}^{2}=0 \% ; \mathrm{OR}, 0.22 ; 95 \%\right.$ Cl, $0.12 \sim 0.40$; Fig. 2B).

\section{Recurrence/ineffectiveness}

Two studies ${ }^{3,16}$ that evaluated recurrence by axilla were excluded from this meta-analysis, and six studies qual-

Table 1. Characteristics of included studies

\begin{tabular}{|c|c|c|c|c|c|c|c|}
\hline Study & Country & Surgery & $\begin{array}{l}\text { No. of } \\
\text { patients }\end{array}$ & $\begin{array}{c}\text { Sex } \\
\text { (male:female) }\end{array}$ & Mean age (yr) & $\begin{array}{l}\text { Follow-up } \\
\text { period (mo) }\end{array}$ & $\begin{array}{l}\text { Quality } \\
\text { score* }\end{array}$ \\
\hline \multirow[t]{2}{*}{ Matsuda $(2004)^{11}$} & \multirow[t]{2}{*}{ Japan } & Open & 64 & $18: 46$ & $28(14 \sim 61)$ & \multirow[t]{2}{*}{$3 \sim 36$} & \multirow[t]{2}{*}{6} \\
\hline & & Closed & 77 & $20: 57$ & $27(14 \sim 69)$ & & \\
\hline \multirow[t]{2}{*}{ Li et al. $(2009)^{12}$} & \multirow[t]{2}{*}{ China } & Open & 52 & \multirow[t]{2}{*}{$N R$} & \multirow[t]{2}{*}{$16 \sim 42$} & \multirow[t]{2}{*}{6 or longer } & \multirow[t]{2}{*}{3} \\
\hline & & Closed & 159 & & & & \\
\hline \multirow[t]{2}{*}{ Li et al. $(2010)^{13}$} & \multirow[t]{2}{*}{ China } & Open & 180 & $125: 55$ & \multirow[t]{2}{*}{ NR } & \multirow[t]{2}{*}{$3 \sim 24$} & \multirow[t]{2}{*}{4} \\
\hline & & Closed & 120 & $47: 73$ & & & \\
\hline \multirow[t]{2}{*}{ Zhang and Yu (2014) } & \multirow[t]{2}{*}{ China } & Open & 40 & $17: 23$ & $22.5(18 \sim 37)$ & \multirow[t]{2}{*}{6 or longer } & \multirow[t]{2}{*}{6} \\
\hline & & Closed & 40 & $18: 22$ & $22.7(18 \sim 36)$ & & \\
\hline \multirow[t]{2}{*}{ Wang et al. $(2015)^{3}$} & \multirow[t]{2}{*}{ China } & Open & 65 & $23: 42$ & $22.9(16 \sim 39)$ & $3 \sim 20$ & \multirow[t]{2}{*}{5} \\
\hline & & Closed & 65 & $23: 42$ & $22.8(15 \sim 38)$ & $3 \sim 40$ & \\
\hline \multirow[t]{2}{*}{ Nam et al. $(2015)^{15}$} & \multirow[t]{2}{*}{ Korea } & Open & 54 & $12: 42$ & 28.1 (NR) & \multirow[t]{2}{*}{19.5} & \multirow[t]{2}{*}{4} \\
\hline & & Closed & 52 & $20: 32$ & 25.9 (NR) & & \\
\hline \multirow[t]{2}{*}{$\mathrm{Li}$ and $\mathrm{Su}(2015)^{16}$} & \multirow[t]{2}{*}{ China } & Open & 45 & $N R$ & \multirow[t]{2}{*}{ NR } & \multirow[t]{2}{*}{6} & \multirow[t]{2}{*}{4} \\
\hline & & Closed & 45 & & & & \\
\hline \multirow[t]{2}{*}{ Hu et al. $(2019)^{17}$} & \multirow[t]{2}{*}{ China } & Open & 60 & $11: 49$ & $22.5(17 \sim 32)$ & \multirow[t]{2}{*}{6} & \multirow[t]{2}{*}{5} \\
\hline & & Closed & 61 & $16: 45$ & $23.7(16 \sim 39)$ & & \\
\hline
\end{tabular}

Values are presented as number only, mean (range), or range. NR: not reported. *Quality was assessed with a modified Newcastle-Ottawa Scale (maximum score of 7 ). High quality was defined as a score of $\geq 5$, while low quality was defined as a score of $\leq 4$. 
A

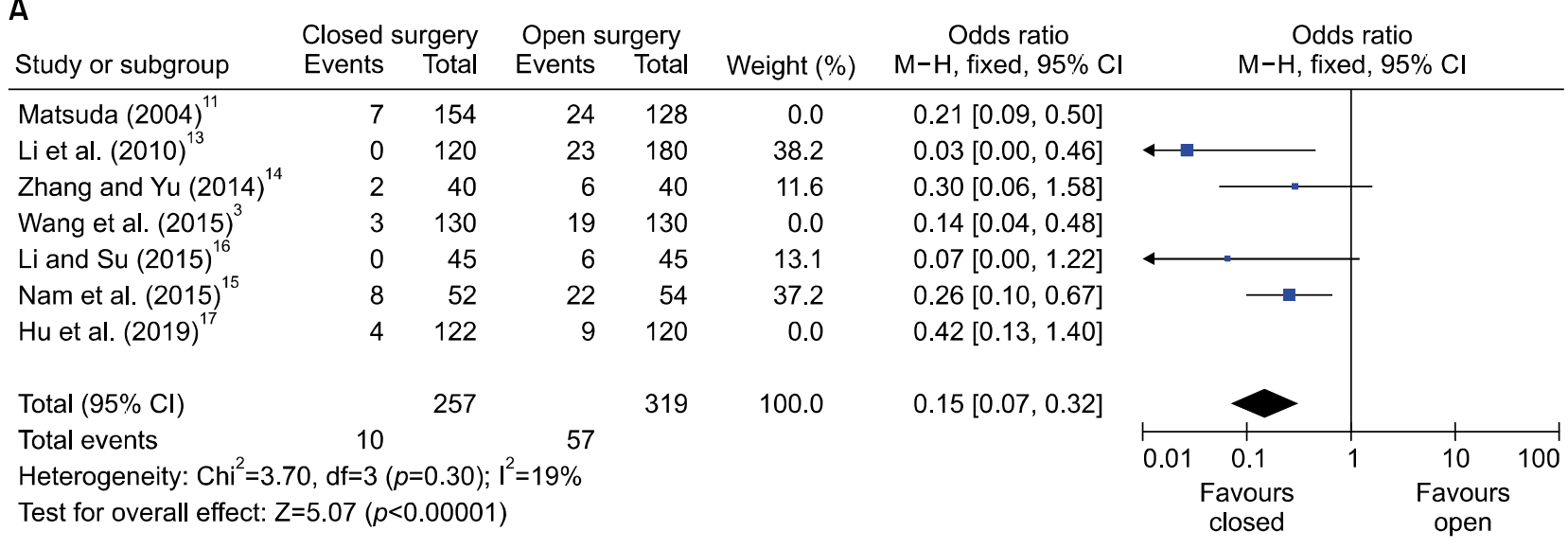

B

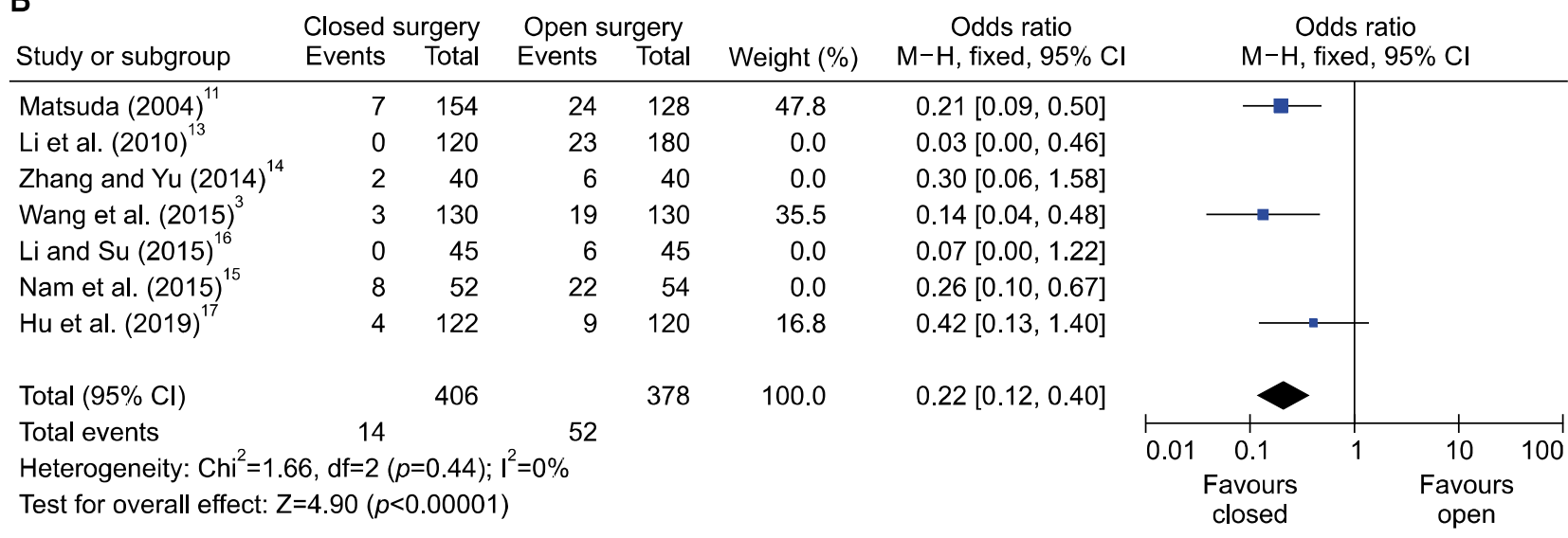

Fig. 2. Forest plots of acute adverse events in open and closed surgeries. Evaluated by individual (A) and evaluated by axilla (B). In both subgroups, open surgery was associated with a significantly greater risk of acute adverse events. M-H: Mantel-Haenszel method, 95\% Cl: confidence intervals.

itatively synthesized in the systematic review were included to determine efficacy. As shown in Fig. $3 A^{11-14,17}$, there was low heterogeneity with respect to recurrence/ineffectiveness, as reflected by an $\mathrm{I}^{2}$ of $33 \%$. The meta-analysis revealed that open excision was significantly superior to suction-curettage in terms of the efficacy (OR, 2.90; 95\% $\mathrm{Cl}, 1.37 \sim 6.15)$. However, when only studies including patients observed for a minimum 3 months were analyzed separately, the recurrence/ineffectiveness rate was not significantly different between open and closed surgeries $\left(\mathrm{I}^{2}=0 \%\right.$; OR, 2.06; 95\% Cl, 0.72 5.93; Fig. 3B) ${ }^{11-14,17}$.

As another indicator of efficacy, patient satisfaction was also compared. Three studies reported that most patients were satisfied with the outcomes of both treatments; $88 \%$ to $98 \%$ in the closed surgery group and $78 \%$ to $98 \%$ in the open surgery group (Fig. 4$)^{3,11,15}$. There was no significant difference in patient satisfaction between the two types of surgery (OR, 1.58; 95\% Cl, 0.69 3.60).

\section{DISCUSSION}

As mentioned in the Introduction section, there have been two meta-analyses of the treatment of axillary osmidrosis in the English literature ${ }^{5,6}$. Shin et al. ${ }^{5}$ concluded that liposuction and open surgery resulted in nearly equivalent rates of complications. However, virtually all studies included in their systematic review and meta-analysis were case series or uncontrolled observations that focused on a single treatment, such as liposuction, open excision, or laser therapy. Zhang et al. ${ }^{6}$ conducted a meta-analysis of patients with osmidrosis treated by suction curettage as an experimental group and concluded that patients who underwent suction curettage had fewer complications than the control group; however, treatments for the control group included various types of open surgery and nonsurgical treatments, such as lasers.

Our recent literature search using PubMed found that more than $90 \%$ of the English-language articles on axillary osmidrosis were from East Asian countries; more than 
A

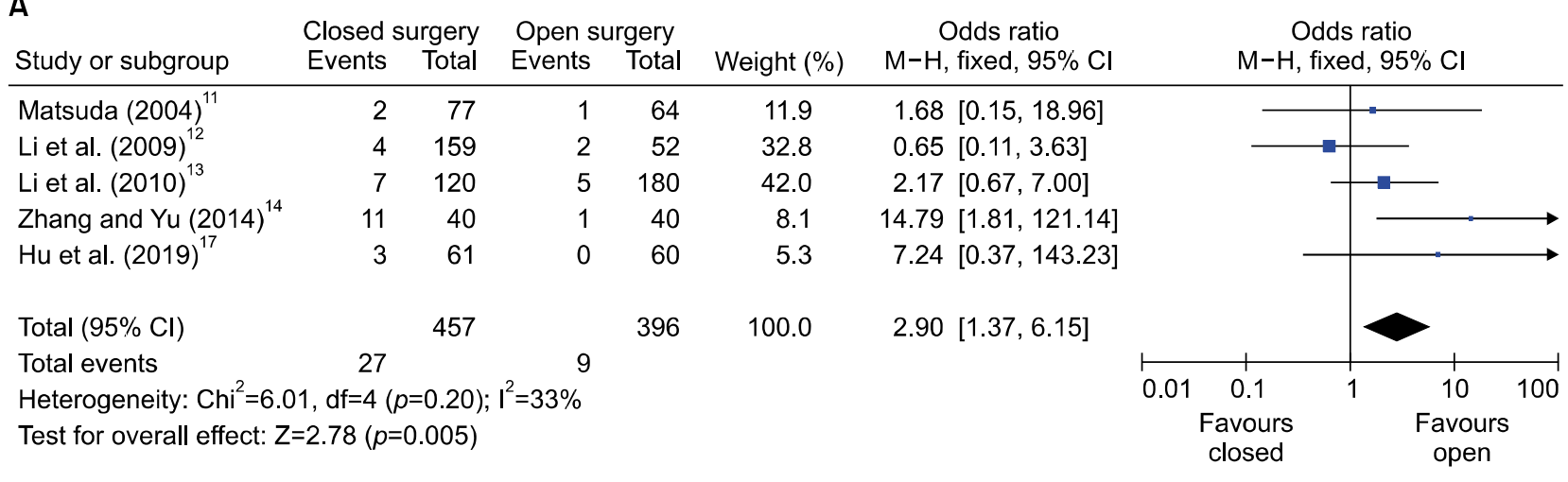

B

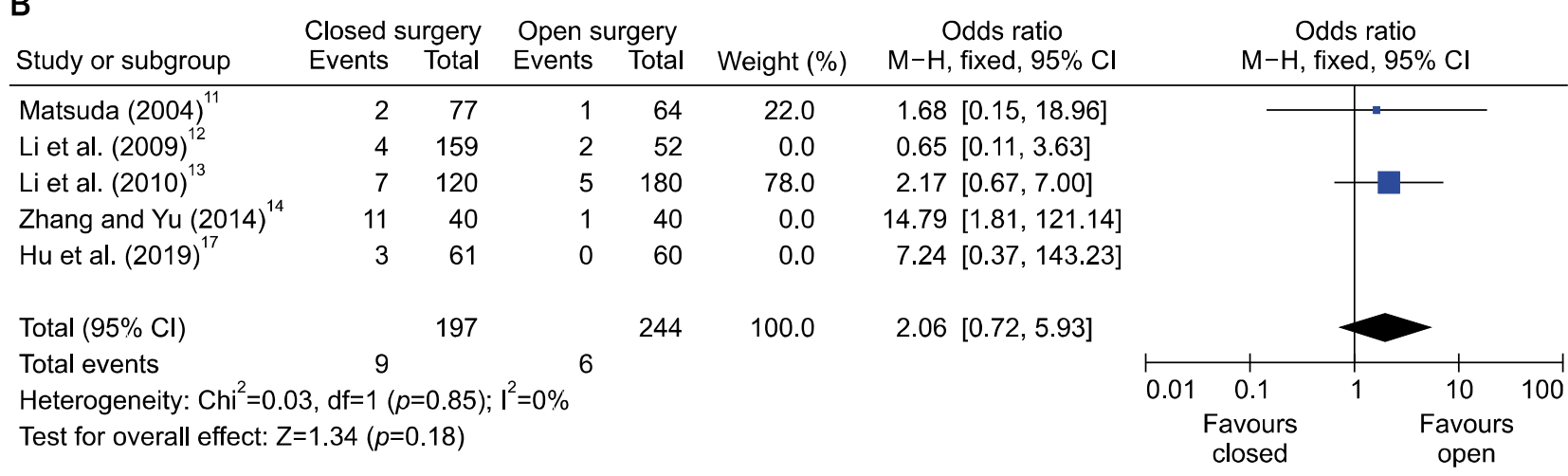

Fig. 3. Forest plot of recurrence/ineffectiveness in open and closed surgeries (A). When the meta-analysis included only studies of patients followed-up for 3 months postoperatively, there was no significant difference in recurrence/ineffectiveness rate between open and closed surgeries (B). M-H: Mantel-Haenszel method, 95\% Cl: confidence intervals.

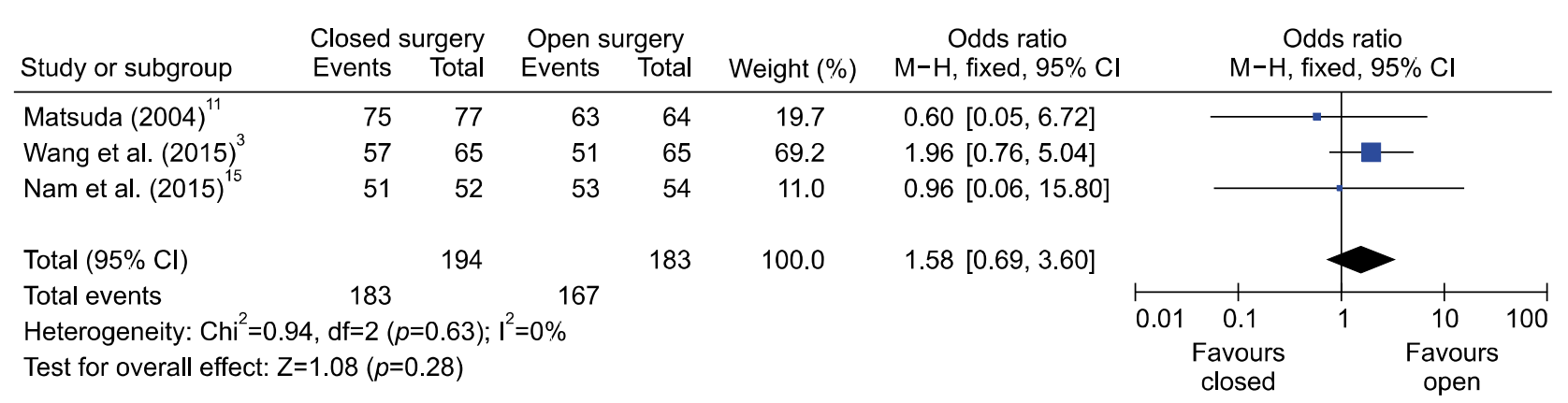

Fig. 4. Forest plot of patient satisfaction with open and closed surgeries. $\mathrm{M}-\mathrm{H}$ : Mantel-Haenszel method, 95\% Cl: confidence intervals.

$30 \%$ were from China, $20 \%$ were from Japan, and $20 \%$ were from Korea. Therefore, the present systematic review was unique in that literature searching was performed in four different languages. The numbers of identified articles in the Japanese (Ichushi-Web=325) and Chinese $(\mathrm{CNKI}=660)$ databases were much greater than in the international databases (PubMed $=110$ and Scopus $=188$ ). Only one article included in the meta-analysis was in English, out of the eight identified through the present systematic review. Results of this meta-analysis did not support those of the previous meta-analysis by Shin et $\mathrm{al}^{5}$. This may be be- cause we included non-English studies that focused on comparisons between open and closed surgeries.

As surgical treatments for axillary osmidrosis have been performed mostly in East Asian societies ${ }^{2}$, it was meaningful to review articles published in three Asian languages. Morrison et al. ${ }^{18}$ stated that the exclusive reliance on English-language studies may not represent all of the evidence: excluding non-English languages may introduce a language bias, and lead to erroneous conclusions. We believe that inclusion of multilingual studies enabled us to conduct a more accurate meta-analysis. 


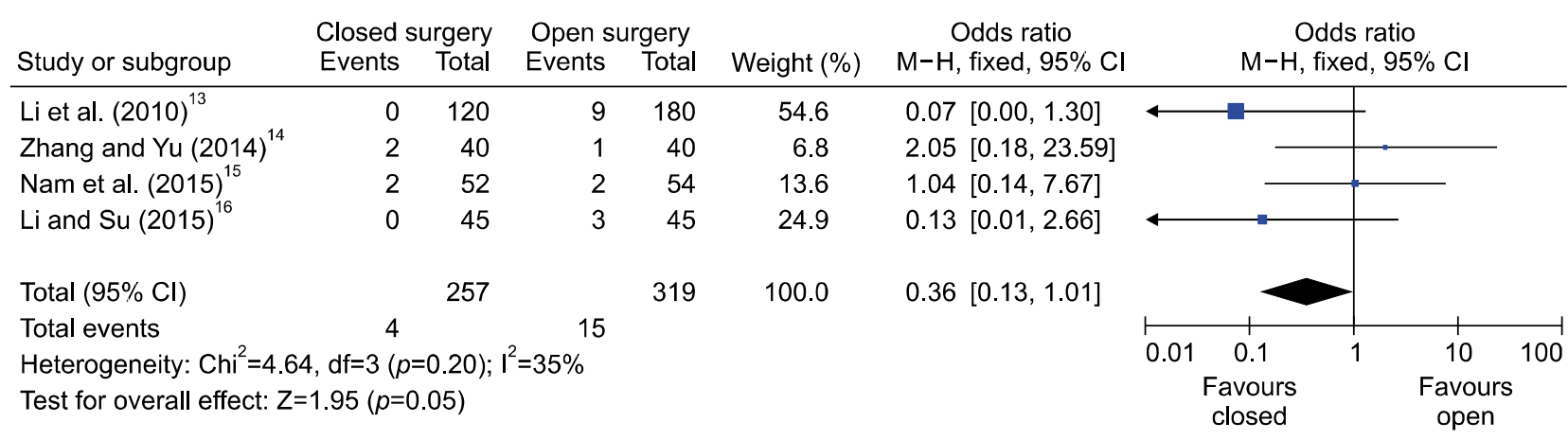

Fig. 5. Forest plot of hematoma occurrence in open and closed surgeries. Open surgery tended to be associated with a greater risk of hematoma, but the result was not statistically significant. $\mathrm{M}-\mathrm{H}$ : Mantel-Haenszel method, $95 \% \mathrm{Cl}$ : confidence intervals.

Our results indicated suction-curettage was more favorable than open excision in terms of safety. In this metaanalysis, we excluded long-lasting and delayed complications from assessments of the safety of surgery, because follow-up periods varied among studies; thus, we assessed safety using the incidence of acute adverse events. Major acute adverse events included hematoma, skin necrosis and wound dehiscence. Although the incidence of hematoma was fairly similar between open and closed surgeries, as shown in Fig. $5\left(\mathrm{I}^{2}=35 \%\right.$; OR, 0.36; $95 \% \mathrm{Cl}$, $0.13 \sim 1.01)^{13-16}$, skin necrosis occurred more frequently in open surgery, and wound dehiscence occurred only in open surgery. We speculate that acute complications were associated more with the interruption of the subdermal plexus by the skin incision than with the method of hemostasis.

In the present meta-analysis, at first, efficacy was evaluated as a low incidence of recurrence/ineffectiveness. Of the eight included studies, three studies ${ }^{3,12,16}$ reported recurrence rates, and five $\mathrm{e}^{11,13-15,17}$ reported ineffectiveness rates; both outcomes were similar in nature, resulting from the remaining apocrine glands. If the remaining glands are transiently denervated by undermining the axillary skin, axillary odor can recur. If they are not denervated during surgery, axillary odor will not improve (ineffective). However, methods to assess such outcomes varied among studies. Matsuda ${ }^{11}$ and Li et al. ${ }^{13}$ assessed efficacy by consensus between physician and patient. Wang et al. ${ }^{3}$ assessed it by smelling the axilla directly or using the physician's finger. Among studies excluded from this systematic review, physicians often used a gauze test to compare the preoperative condition and the postoperative outcome ${ }^{19}$. However, these objective tests are influenced by several factors, such as the patient's clothes and hygiene, room temperature, and the rater's sense of smell ${ }^{1}$.

Hence, it might be more important to investigate patients' satisfaction as an indicator to assess efficacy of treatments for axillary osmidrosis ${ }^{3,19}$. Huang et al. ${ }^{20}$ proposed the use of a dermatology life quality index for postoperative evaluation of the treatment. Wang et al. ${ }^{3}$ used a face scale. Nam et al. ${ }^{15}$ used a patient questionnaire. Most methods were an evaluation in view of patients themselves for the total treatment program including the procedure, discomfort of dressing, recovery time, outcomes, scarring, and cost. In this systematic review, three studies ${ }^{3,11,15}$ investigated patient satisfaction. Interestingly, both patient groups were highly satisfied with the treatments, and the meta-analysis demonstrated no significant difference in patient satisfaction between open and closed surgeries. We propose that all future studies on the treatment for axillary osmidrosis quantify patient satisfaction using some scale. Several modifications of open excision surgery have been reported, which include multiple incisions ${ }^{21}$ and combination with partial skin excision ${ }^{22}$. However, only studies that used conventional open excision through a single skin incision were included in this meta-analysis. In contrast, a variety of suction-curettage procedures have been used for closed surgery, which includes the curette provided with the suction system ${ }^{23}$, a Fatemi/Cassio cannu$\mathrm{la}^{16}$, and a cartilage-shaver system ${ }^{1,11,20}$.

It was difficult to compare safety and efficacy among these modifications, because most reports were non-controlled, clinical observations. Although only Matsuda ${ }^{11}$ used a cartilage-shaver system in this meta-analysis, other surgeons $^{24,25}$ who used the same system for axillary osmidrosis reported that recurrence rates were also very low $(0 \% \sim 2.6 \%)$ compared to other suction-curettage techniques.

We have treated more than 600 individuals with axillary osmidrosis using either open excision or suction-curettage with a cartilage-shaver system ${ }^{1}$. Our preliminary, ongoing study on a retrospective cohort of these two treatments revealed that the complication rate of the cartilage-shaver system was much lower than that of open excision, where- 
as the recurrence rate was as low as that of open surgery (approximately $2 \%$ ) [unpublished data].

Recently, a few technologies have emerged for the treatment of axillary osmidrosis ${ }^{2}$. In particular, the microwave technology had a significantly lower complication rate than suction-curettage in a comparative study ${ }^{26}$. This procedure is nonsurgical, probably much safer than surgery, and may potentially be permanent, although long-term recurrence rates were unclear. If such new technologies were performed as commonly as conventional treatments, a meta-analysis with respect to long-term outcomes would be required in the future.

Our results should be interpreted in the context of a few limitations. First, as shown in Fig. $2^{3,11,13-17}$, three studies counted the number of adverse events by axilla, and four counted the number of adverse events by patient, so we had to analyze them separately. Furthermore, it is possible that two or more adverse events that could be related to one another (e.g. skin necrosis resulting from hematoma) occurred among patients included in this meta-analysis. Second, in three studies ${ }^{3,11,13}$, the minimum follow-up period was 3 months. As shown in Fig. $5^{13-16}$, the meta-analysis including only these three studies did not demonstrate a significant difference in the recurrence/ineffectiveness rate between open and closed surgeries. Wang et al. ${ }^{3}$ concluded that it might be too early to assess final efficacy results at 3 months after surgery. Several authors, including us, have encountered axillary odor that recurred 6 months or later after surgery ${ }^{3,11,26}$.

Thus, it is important to standardize how to assess complications and outcomes. We propose that both should be assessed by individual rather than by axilla, and efficacy should be assessed at a minimum of 6 months after surgery. In summary, the four-language database search yielded 8 potentially eligible articles: 5 in Chinese, 1 in English, 1 in Japanese, and 1 in Korean. Our clinical question was, "Which surgical method, open excision and suction-curettage, is more favorable in terms of safety and efficacy for the treatment of axillary osmidrosis?" The answer suggested by this analysis was that open surgery was more effective but less safe than closed surgery. However, both patient groups expressed very high satisfaction with each treatment approach. Because objective odor tests are influenced by the rater and the patient's condition and circumstances, patient satisfaction scales should be used to evaluate treatment efficacy.

Treatment options for axillary osmidrosis cannot be determined based only on safety and efficacy; other factors, such as cost, postoperative discomfort, recovery time, and scar formation, should also be considered. Compared to open surgery, closed surgery is not cost-effective, but it is advantageous in terms of a short recovery time and low complication rate ${ }^{1}$. Cost may be a large factor when patients are making treatment decisions ${ }^{27}$. In addition, emerging technologies have already demonstrated fair effects with very few complications ${ }^{26}$, but are disadvantageous in terms of financial factors. Further comparative studies between emerging treatment options will be necessary so that we can recommend the best treatment options for axillary osmidrosis to our patients.

\section{CONFLICTS OF INTEREST}

The authors have nothing to disclose.

\section{FUNDING SOURCE}

None.

\section{DATA SHARING STATEMENT}

Research data are not shared.

\section{ORCID}

Misako Nomura, https://orcid.org/0000-0002-1816-9926

Daichi Morioka, https://orcid.org/0000-0002-6210-9808

Yasutaka Kojima, https://orcid.org/0000-0002-4806-8667

Ryutaro Tanaka, https://orcid.org/0000-0003-2720-3097

Koichi Kadomatsu, https://orcid.org/0000-0002-8632-9389

\section{REFERENCES}

1. Morioka D, Ohkubo F, Amikura Y. Clinical features of axillary osmidrosis: a retrospective chart review of 723 Japanese patients. J Dermatol 2013;40:384-388.

2. Morioka D, Nomura M, Lan L, Tanaka R, Kadomatsu K. Axillary osmidrosis: past, present, and future. Ann Plast Surg 2020;84:722-728.

3. Wang $\mathrm{C}, \mathrm{Wu} \mathrm{H}$, Du F, Le S, Zheng S. Axillary osmidrosis treatment using an aggressive suction-curettage technique: a clinical study on paired control. Aesthetic Plast Surg 2015; 39:608-615.

4. Jung SK, Jang HW, Kim HJ, Lee SG, Lee KG, Kim SY, et al. A prospective, long-term follow-up study of 1,444 nm Nd:YAG laser: a new modality for treating axillary bromhidrosis. Ann Dermatol 2014;26:184-188.

5. Shin JY, Roh SG, Lee NH, Yang KM. Osmidrosis treatment approaches: a systematic review and meta-analysis. Ann Plast Surg 2017;78:354-359.

6. Zhang L, Chen F, Kong J, Li Z, Wen X, Liu J. The curative effect of liposuction curettage in the treatment of bromhidrosis: a meta-analysis. Medicine (Baltimore) 2017;96:e7844.

7. Inaba M, Ezaki T. New instrument for hircismus and 
hyperhidrosis operation: subcutaneous tissue shaver. Plast Reconstr Surg 1977;59:864-866.

8. Ou LF, Yan RS, Chen IC, Tang YW. Treatment of axillary bromhidrosis with superficial liposuction. Plast Reconstr Surg 1998;102:1479-1485.

9. Moher D, Liberati A, Tetzlaff J, Altman DG; PRISMA Group. Preferred reporting items for systematic reviews and metaanalyses: the PRISMA statement. BMJ 2009;339:b2535.

10. Oteng-Ntim E, Mononen S, Sawicki O, Seed PT, Bick D, Poston L. Interpregnancy weight change and adverse pregnancy outcomes: a systematic review and meta-analysis. BMJ Open 2018;8:e018778.

11. Matsuda K. Surgical treatment of axillary osmidrosis with the shaver system. Jpn J Plast Reconstr Surg 2004;47:12531259.

12. Li BW, Tao HW, Wang MJ. Comparision of three methods in treatment of osmidrosis. Chin J Med Aesthet Cosmetol 2009;15:107-109.

13. Li WW, Liu ZF, Cui YN, Zeng A. [Comparison study on the efficacy of three methods for the treatment of osmidrosis]. Zhonghua Zheng Xing Wai Ke Za Zhi 2010;26:348-350. Chinese.

14. Zhang JZ, Yu L. Comparison among two small incision procedures in treating axillary osmidrosis. China Med Her 2014;(26):62-64.

15. Nam SH, Lee JY, Kim SS, Kim CW, Min HG. Comparison between conventional and modified superficial liposuction techniques in the treatment of axillary osmidrosis. Korean J Dermatol 2015;53:523-529.

16. Li L, Su BX. Comparative study on the efficacy of two different micro-invasive treatment for axillary bromhidrosis. Chin J Aesthetic Med 2015;24:17-19.

17. Hu RL, Guo Y, Long JH. Comparison of the clinical efficacy of subcutaneous pruning combined with Xiaozhiling infusion and negative pressure suction combined with Xiaozhiling perfusion in the treatment of axillary odor. Chin J Aesthet Plast Surg 2019;30:28-31.

18. Morrison A, Polisena J, Husereau D, Moulton K, Clark M, Fiander $M$, et al. The effect of English-language restriction on systematic review-based meta-analyses: a systematic review of empirical studies. Int J Technol Assess Health Care 2012; 28:138-144.

19. Niiyama $S$, Aiba $S$, Katsuoka $K$, Ito $Y$, Sumiya N. Treatment of osmidrosis using the ultrasonic surgical aspirator. Acta Derm Venereol 2006;86:238-240.

20. Huang $\mathrm{YH}$, Yang $\mathrm{CH}$, Chen $\mathrm{YH}$, Chen $\mathrm{CH}$, Lee $\mathrm{SH}$. Reduction in osmidrosis using a suction-assisted cartilage shaver improves the quality of life. Dermatol Surg 2010;36: 1573-1577.

21. Li ZR, Sun CW, Zhang JY, Qi YQ, Hu JZ. Excision of apocrine glands with preservation of axillary superficial fascia for the treatment of axillary bromhidrosis. Dermatol Surg 2015;41:640-644.

22. Wu WH, Ma S, Lin JT, Tang YW, Fang RH, Yeh FL. Surgical treatment of axillary osmidrosis: an analysis of 343 cases. Plast Reconstr Surg 1994;94:288-294.

23. Shi Z, Yan X, Ye X. Modified tumescent superficial suction with curettage treatment for axillary bromidrosis: clinical experience of 280 cases. Aesthetic Plast Surg 2014;38:151155.

24. Lee JC, Kuo HW, Chen $\mathrm{CH}$, Juan $\mathrm{WH}$, Hong $\mathrm{HS}$, Yang $\mathrm{CH}$. Treatment for axillary osmidrosis with suction-assisted cartilage shaver. Br J Plast Surg 2005;58:223-227.

25. Wu WH. Ablation of apocrine glands with the use of a suction-assisted cartilage shaver for treatment of axillary osmidrosis: an analysis of 156 cases. Ann Plast Surg 2009; 62:278-283.

26. Yang $\mathrm{HH}$, Miao $\mathrm{Y}, \mathrm{Chen} \mathrm{YT}, \mathrm{Hu} Z \mathrm{ZQ}$. Minimally invasive approaches to axillary osmidrosis treatment: a comparison between superficial liposuction with automatic shaver curettage, subcutaneous laser treatment, and microwave-based therapy with a modified technique. J Cosmet Dermatol 2019; 18:594-601.

27. Liu Q, Zhou Q, Song Y, Yang S, Zheng J, Ding Z. Surgical subcision as a cost-effective and minimally invasive treatment for axillary osmidrosis. J Cosmet Dermatol 2010;9: 44-49. 